\title{
SYMMETRY OF SOLITARY WATER WAVES WITH VORTICITY
}

\author{
Vera Mikyoung Hur
}

\begin{abstract}
Symmetry and monotonicity properties of solitary water-waves of positive elevation with supercritical values of parameter are established for an arbitrary vorticity. The proof uses the detailed knowledge of asymptotic decay of supercritical solitary waves at infinity and the method of moving planes.
\end{abstract}

\section{Introduction}

A priori geometric properties are considered for exact free-surface water waves which steadily propagate of permanent form over a shear flow of finite depth. The main result states that for an arbitrary distribution of vorticity any solitary wave of positive elevation and with a supercritical value of parameter* is symmetric about a single crest on either side of which the wave profile decreases monotonically to its asymptotic level of height. The proof relies on the method of moving planes as adapted for a nonlinear elliptic boundary value problem in an infinite horizontal strip. The asymptotic decay properties of solitary waves to the background shear flow are derived.

Particular emphasis is on the effects of vorticity. While the irrotational assumption ${ }^{\dagger}$ under certain circumstances may serve as an approximation in the study of the waterwave problem and it has been used in a majority of the existing research, waves on the surface of water typically carry vorticity, e.g. wind-drift boundary layers and shear currents on a shallow channel. Moreover, the governing equations for water waves allow for rotational motions.

For an arbitrary vorticity distribution demonstrated in [13] is the existence of supercritical solitary waves of small amplitude, which decay exponentially away from their principal elevation. The construction in [13] assumes that the wave profiles are symmetric; the present investigation is their a priori symmetry and monotonicity properties. Our method is free from restrictions on wave amplitude, and as such

Received by the editors May 9, 2007.

2000 Mathematics Subject Classification. Primary: 76B15, Secondary:74J35, 35J65, 35B50, 35B40.

Key words and phrases. water waves, solitary, vorticity, symmetry, moving planes, maximum principles.

*Supercritical values of parameter are those for which zero does not lie in the continuous spectrum of the corresponding linearized problem. Its definition is given in Section 2.

${ }^{\dagger}$ The irrotational water-wave problem reduces to one of potential flows with its nonlinearity only at the boundary conditions, and as such its analysis often uses special techniques of complex analysis or harmonic analysis. The rotational problem, on the other hand, is intricate due to the additional nonlinearity in the field equation. 
rotational solitary waves of large amplitude, if exist, must have a symmetric and monotone profile.

In the irrotational setting, the mathematical existence theory for solitary waterwaves dates back to the construction due to Friedrichs and Hyers [6] and Beale [2] of small-amplitude waves, and it includes the work of Amick and Toland [1] on the global bifurcation result of large-amplitude waves. Craig and Sternberg $[4,5]$ demonstrated the symmetry, monotonicity and positivity properties of solitary waves of the irrotational water-wave problem as well as other potential-flow problems with free surface. Keady and Pritchard [14] proved that solitary waves which are symmetric and monotone are possible only when the Froude number is greater than one, that is, the parameter values are supercritical, and when the wave profile is positively elevated. McLeod [16] derived the same supercritical-ness result and showed that the total mass above the asymptotic height is finite.

Our symmetrization analysis in Section 3 is based on the maximum principle and a device of moving parallel planes to a critical position and then showing that the solution is symmetric about the limiting plane. This so-called method of moving planes was initially developed due to Alexandrov [11, Chap. 7] and was refined extensively in the works due to Serrin [17], Gidas, Ni and Nirenberg [7] and many others.

In order to initiate the method of moving planes in an unbounded domain, in general, it is prerequisite to have precise knowledge on the behavior of solutions at infinity. Section 4 is devoted to the asymptotic description of the wave profile in the far field of solitary water-waves with vorticity. Our result is closely related to that in $[4,5]$. First, it is shown that any solitary-wave solution with a supercritical value of parameter, which tends to the asymptotic level of height at infinity, does at an exponential rate. The decay rate is measured by the (positive) beginning value of the continuous spectrum of the corresponding linear operator. Secondly, an asymptotic expansion of solutions at infinity is obtained.

Recorded in Section 2 are the vorticity-stream formulation of the solitary waterwave problem as a stationary elliptic problem with free surface and its reformulation as a nonlinear elliptic boundary value problem in a fixed infinite strip.

The positivity property of free-surface elevation and supercritical-ness of parameter value in the statement of our result are hypotheses while in the irrotational setting they are conclusions of a priori analysis. Indeed, by a simple comparison principle follows the positivity property of surface elevation of irrotational solitary waves [4], and by inspecting Bernoulli's law follows the supercritical-ness of parameter value of symmetric solitary waves with zero vorticity $[14,16]$. It is of great interest to study these properties and other geometric properties of solitary waves for a general class of vorticities.

Our moving-plane technique applies to a general class of elliptic problems in domains which possess translational symmetry. In particular, it is established in [12] that under certain conditions on the streamline pattern any periodic water-wave with vorticity is symmetric and monotone. Combined with a connectedness argument, this asserts that all periodic-wave solutions constructed in [3], which are in a (connected) solution branch containing small-amplitude solutions with the desired streamline pattern, are symmetric and monotone (Remark 3.2). 


\section{Formulations}

2.1. The vorticity-stream formulation for steady water-waves. We concern with steady-wave solutions of the Euler equations in a horizontal channel of an incompressible inviscid fluid with a free surface, acted on by gravity. The effects of surface tension are neglected. Suppose, for definiteness, that in the $(x, y)$-Cartesian coordinates the gravity acts in the negative $y$-direction. Suppose that, in the frame of reference moving at the speed of wave propagation, the liquid occupies the stationary region

$$
\{(x, y):-\infty<x<\infty,-d<y<\eta(x)\},
$$

where $0<d<\infty$ is the asymptotic depth of the channel. Let $\psi(x, y)$ denote the stream function associated with the flow, and as such $\left(\psi_{y},-\psi_{x}\right)$ represents the stationary velocity field. The flow is allowed to be rotational and characterized by the vorticity, denoted by $\omega(x, y)$. By definition, then, $\omega=-\Delta \psi$. The steady water-wave problem states as follows [3, Section 2], [13, Section 2]:

For a function $\gamma \in C^{1+\alpha}\left(\left[0,\left|p_{0}\right|\right]\right)$, where $\alpha \in(0,1)$, there exist a real parameter $\lambda$ and functions $\psi(x, y)$ of class $C^{2+\alpha}$ and $\eta(x) \in C^{3+\alpha}(\mathbb{R})$ such that

$$
\begin{array}{cl}
-\Delta \psi=\gamma(\psi) & \text { in } \quad-d<y<\eta(x), \\
\psi=0 & \text { on } \quad y=\eta(x), \\
|\nabla \psi|^{2}+2 g y=\lambda & \text { on } \quad y=\eta(x), \\
\psi=-p_{0} & \text { on } \quad y=-d,
\end{array}
$$

and, in addition,

$$
\psi_{y}<0 \quad \text { in } \quad-d<y<\eta(x),
$$

where

$$
p_{0}=\int_{-d}^{\eta(x)} \psi_{y}(x, y) d y
$$

is the relative total flux ${ }^{\ddagger}$.

The solitary-wave problem is (2.1a)-(2.1e) supplemented with the asymptotic boundary conditions at infinity

$$
\begin{cases}\eta(x) \rightarrow 0 & \text { as } \quad|x| \rightarrow \infty \\ \psi_{x}(x, y) \rightarrow 0 & \text { as } \quad|x| \rightarrow \infty \text { uniformly for } y\end{cases}
$$

which express respectively that in the far field the free surface approaches the asymptotic level $y=0$ above the bottom of the channel and that the flow motion is nearly horizontal.

The vorticity function $\gamma(\psi)$ in $(2.1 \mathrm{a})$ gives the relation between the vorticity and the stream function, that is, $\omega=\gamma(\psi)$. The so-called assumption of no criticallayers $^{\S}(2.1 \mathrm{e})$ guarantees that such a function is well-defined throughout the fluid

\footnotetext{
${ }^{\ddagger} p_{0}<0$ is independent of $x$; see [3], for instance.

${ }^{\S}$ In other words, there is no stagnation in the fluid region. Field observations [15] as well as laboratory experiments [19] indicate that for wave patterns which are not near the spilling or breaking state, the speed of wave propagation is in general considerably larger than the horizontal velocity of any water particle.
} 
region; see [3]. Let

$$
\Gamma(p)=\int_{0}^{p} \gamma(-s) d s \quad \text { and } \quad \Gamma_{\min }=\min _{\left[p_{0}, 0\right]} \Gamma(p) \leqslant 0 .
$$

The nonlinear boundary condition (2.1c) at the free surface expresses Bernoulli's law. In view of (2.1f) the parameter $\lambda$ is the square of the upstream flow speed in the far field, i.e.,

$$
\psi_{y}^{2}(x, \eta(x)) \rightarrow \lambda \quad \text { as } \quad|x| \rightarrow \infty .
$$

Hence, $\lambda>0$. The critical value $\lambda_{c}$ of $\lambda$ is [13] the unique solution of

$$
\int_{p_{0}}^{0} \frac{d p}{{\sqrt{\lambda_{c}+2 \Gamma(p)}}^{3}}=\frac{1}{g}
$$

for which zero is at the beginning of the continuous spectrum of the linearized problem around the background shear flow; see Lemma 4.1.

The existence of small-amplitude solutions of (2.1) for an arbitrary $\gamma \in C^{1+\alpha}\left(\left[0,\left|p_{0}\right|\right)\right.$ is recently established in [13] under the condition that the parameter value is supercritical, i.e. $\lambda>\lambda_{c}$, but close to $\lambda_{c}$. In the irrotational setting, i.e., $\gamma \equiv 0$, Keady and Pritchard [14] proved that symmetric and monotone solitary waves are possible only for supercritical ${ }^{\mathbb{\pi}}$ values of parameter $\lambda>\lambda_{c}$ and $\eta(x)>0$ for $x \in \mathbb{R}$. McLeod [16] derived the same result and showed that the total mass of the fluid is finite, that is, $\int_{-\infty}^{\infty} \eta(x) d x<\infty$.

2.2. Reformulation: reduction to the nonlinear elliptic boundary value problem. Under the assumption (2.1e) interchanging the roles of the $y$-coordinate and $\psi$ offers an alternative formulation to (2.1) in a fixed infinite strip, which is now presented. A detailed discussion is found in [13, Section 3].

Let us define the new independent variables as

$$
q=x \text { and } p=-\psi(x, y),
$$

which map the fluid region $\{(x, y):-\infty<x<\infty,-d<y<\eta(x)\}$ in the $(x, y)$ plane onto the infinite strip $(-\infty, \infty) \times\left(p_{0}, 0\right)$ in the $(q, p)$-plane and the wave profile $y=\eta(x)$ onto the horizontal line $p=0$. Correspondingly, the vertical elevation measured from the flat bottom $h(q, p)=y+d$ replaces the dependent variable. An explicit calculation yields that

$$
h_{q}=-\frac{\psi_{x}}{\psi_{y}}, \quad h_{p}=-\frac{1}{\psi_{y}} .
$$

It is straightforward that the Poisson equation (2.1a) in the transformed variables becomes

$$
h_{p}^{2} h_{q q}-2 h_{q} h_{p} h_{q p}+\left(1+h_{q}^{2}\right) h_{p p}=-\gamma(-p) h_{p}^{3} \quad \text { in } \quad p_{0}<p<0,
$$

and that Bernoulli's law (2.1c) and the bottom boundary condition (2.1d), respectively, translate into

$$
\begin{array}{cl}
1+h_{q}^{2}+(2 g h-2 g d-\lambda) h_{p}^{2}=0 & \text { on } p=0, \\
h=0 & \text { on } p=p_{0} .
\end{array}
$$

`It corresponds to that the Froude number is bigger than one. 
The condition of no critical layer (2.1e) states that

$$
h_{p}>0 \text { throughout } p_{0}<p<0 .
$$

Finally, the asymptotic boundary conditions (2.1f) are

$$
\begin{cases}h(q, 0) \rightarrow d & \text { as } \quad|q| \rightarrow \infty, \\ h_{q}(q, p) \rightarrow 0 & \text { as } \quad|q| \rightarrow \infty \quad \text { uniformly for } p .\end{cases}
$$

The above formulation (2.3) is equivalent to (2.1). The proof is nearly identical to that in [3, Lemma 2.1].

In order to conveniently state and prove a priori decay properties of solutions of (2.3), furthermore, let us decompose

$$
h(q, p)=H(p)+w(q, p),
$$

where $H(0)=d$ and $w(q, 0) \rightarrow 0$ as $|q| \rightarrow \infty$, and write (2.3) in terms of $w$. Indeed, an explicit calculation yields that [13, Lemma 1] for $\lambda>-2 \Gamma_{\min } \geqslant 0$, the system (2.3) admits a solution

$$
H(p)=H(p ; \lambda)=\int_{p_{0}}^{p} \frac{d s}{\sqrt{\lambda+2 \Gamma(s)}},
$$

which corresponds to a horizontal shear flow under a flat surface. In view of (2.3b) it follows that

$$
H(0)=\int_{p_{0}}^{0} \frac{d p}{\sqrt{\lambda+2 \Gamma(p)}}=d .
$$

Thus, $w(q, p)$ represents the difference of the vertical elevation of a solitary-wave solution and that of its background flow. Let us introduce the notation

$$
a_{\lambda}(p)=\sqrt{\lambda+2 \Gamma(p)} .
$$

Using it, $H^{\prime}(p)=a_{\lambda}^{-1}(p)$.

Since

$$
\lim _{\lambda \rightarrow-2 \Gamma_{\min }} \int_{p_{0}}^{0} a_{\lambda}^{-3}(p)=\infty \text { and } \lim _{\lambda \rightarrow \infty} \int_{p_{0}}^{0} a_{\lambda}^{-3}(p)=0,
$$

by continuity, there exists a solution $\lambda_{c}>-2 \Gamma_{\min } \geqslant 0$ of (2.2). Moreover, the solution $\lambda_{c}$ is unique. Indeed, the mapping $\lambda \mapsto \int_{p_{0}}^{0} a_{\lambda}^{-3}(p) d p$ is strictly decreasing. Such a unique solution $\lambda_{c}$ is called the critical value of parameter.

Finally, a simple substitution yields the (concise form) of the solitary-wave problem:

For $p_{0}<0$ and $\gamma \in C^{1+\alpha}\left(\left[0,\left|p_{0}\right|\right]\right)$, where $\alpha \in(0,1)$, there exist a real parameter $\lambda>0$ and a function $w(q, p)$ defined in the infinite strip

$$
S:=\left\{(q, p):-\infty<q<\infty, p_{0}<p<0\right\}
$$

such that

$$
\begin{cases}F_{1}\left(\nabla w, \nabla^{2} w\right)=0 & \text { in } S \\ F_{2}(w, \nabla w)=0 & \text { for } p=0 \\ w=0 & \text { for } p=p_{0},\end{cases}
$$


and

$$
\begin{array}{cl}
w, \nabla w, \nabla^{2} w \rightarrow 0 & \text { as }|q| \rightarrow \infty \quad \text { uniformly for } p \\
a_{\lambda}^{-1}+w_{p}>0 & \text { throughout } S
\end{array}
$$

where

$$
\begin{aligned}
F_{1}\left(\nabla w, \nabla^{2} w\right)= & \left(a_{\lambda}^{-1}(p)+w_{p}\right)^{2} w_{q q}-2\left(a_{\lambda}^{-1}(p)+w_{p}\right) w_{q} w_{p q}+\left(1+w_{q}^{2}\right) w_{p p} \\
& -\gamma(-p) a_{\lambda}^{-3}(p)\left(1+w_{q}^{2}\right)+\gamma(-p)\left(a_{\lambda}^{-1}(p)+w_{p}\right)^{3}, \\
F_{2}(w, \nabla w)=1 & +w_{q}^{2}+(2 g w-\lambda)\left(\lambda^{-1 / 2}+w_{p}\right)^{2} .
\end{aligned}
$$

The principal part of $F_{1}$ is denoted by

$$
A(\nabla w)=\left(a_{\lambda}^{-1}(p)+w_{p}\right)^{2} \partial_{q}^{2}-2\left(a_{\lambda}^{-1}(p)+w_{p}\right) w_{q} \partial_{q} \partial_{p}+\left(1+w_{q}^{2}\right) \partial_{p}^{2} .
$$

Clearly, $F_{1}$ and $F_{2}$ are smooth. Note that the differential operator $F_{1}$ is uniformly elliptic. Indeed, the coefficients of the principal part satisfy

$$
\left(a_{\lambda}^{-1}(p)+w_{p}\right)^{2} \xi_{1}^{2}-2\left(a_{\lambda}^{-1}(p)+w_{p}\right) w_{q} \xi_{1} \xi_{2}+\left(1+w_{q}^{2}\right) \xi_{2}^{2} \geqslant 4\left(\inf _{S}\left(a_{\lambda}^{-1}(p)+w_{p}\right)\right)^{2}\left(\xi_{1}^{2}+\xi_{2}^{2}\right)
$$

for all $\left(\xi_{1}, \xi_{2}\right) \in \mathbb{R}^{2}$. Note that the boundary operator $F_{2}$ is uniformly oblique in the sense that it is bounded away from being tangential:

$$
\left|\frac{\partial F_{2}}{\partial w_{p}}\right|=\left|2(2 g w-\lambda)\left(\lambda^{-1 / 2}+w_{p}\right)\right|>2\left(\sup _{p=0}\left(\lambda^{-1 / 2}+w_{p}\right)\right)^{-1}>0 .
$$

\section{Symmetry of solitary waves}

Our goal in this section is the demonstration of the symmetry and monotonicity properties for an arbitrary vorticity of supercritical solitary waves with positive elevation.

The theorem below is a precise statement of the sense in which a solitary wave is symmetric and monotone.

Theorem 3.1. For $p_{0}<0$ and $\gamma \in C^{1+\alpha}\left(\left[0,\left|p_{0}\right|\right]\right)$, where $\alpha \in(0,1)$, let $\lambda>\lambda_{c}$ and $w \in C^{2+\alpha}(\bar{S})$ be a nontrivial solution pair of $(2.6)$ satisfying $w(q, 0)>0$ for all $q \in \mathbb{R}$. Then, $w(q, p)$ is symmetric in the q-variable. That is, for a vertical axis of symmetry $q=\tau^{*}$

$$
w(q, p)=w\left(2 \tau^{*}-q, p\right) \quad \text { for } \quad(q, p) \in \bar{S} .
$$

Moreover, $w(q, p)$ monotonically decreases on either side of $q=\tau^{*}$. That is,

$$
w_{q}(q, p)>0 \quad \text { for } \quad-\infty<q<\tau^{*}, p_{0}<p \leqslant 0 .
$$

We first claim that the assumption that $w(q, 0)>0$ for all $q \in \mathbb{R}$ in Theorem 3.1 implies the weak form of positivity, $w(q, p) \geqslant 0$ in $S$. Indeed, let $w \in C^{2}(\bar{S})$ be as in Theorem 3.1. Note that

$$
F_{1}\left(\nabla w, \nabla^{2} w\right)=A(\nabla w)[w]-\gamma(-p) a_{\lambda}^{-3}(p)\left(1+w_{q}^{2}\right)+\gamma(-p)\left(a_{\lambda}^{-1}(p)+w_{p}\right)^{3}=0
$$

in $S$ and that $w\left(q, p_{0}\right)=0$ and $w \rightarrow 0$ as $|q| \rightarrow \infty$ uniformly for $p$. Since $w(q, 0)>0$ for $q \in \mathbb{R}$, the Phragmén-Lindelöf principle [9] applies to assert the claim. 
To proceed the detailed symmetrization analysis, we need to describe several notations. For $\tau \in \mathbb{R}$ a parameter, let us denote the half strip

$$
S(\tau)=\{(q, p) \in S: q<\tau\} .
$$

The idea of the proof is to compare at $(q, p) \in S(\tau)$ its evaluations under the function $w(q, p)$ and under the reflected function

$$
w^{\tau}(q, p)=w(q, p ; \tau):=w(2 \tau-q, p) .
$$

Let us denote functions defined in $S(\tau)$ as

$$
v^{\tau}(q, p)=v(q, p ; \tau):=w^{\tau}(q, p)-w(q, p), \quad f^{\tau}(q, p)=w^{\tau}(q, p)+w(q, p) .
$$

It is straightforward that $v^{\tau}$ is a solution of the linear elliptic boundary value problem

$$
\begin{aligned}
& L\left[v^{\tau}\right]=A(\nabla w)\left[v^{\tau}\right]+\left[w_{p p}^{\tau} f_{q}^{\tau}-2\left(a_{\lambda}^{-1}(p)+w_{p}^{\tau}\right) w_{p q}^{\tau}-\gamma(-p) a_{\lambda}^{-3}(p) f_{q}^{\tau}\right] v_{q}^{\tau} \\
& +\left[-2 w_{q} w_{p q}^{\tau}+\left(2 a_{\lambda}^{-1}(p)+f_{p}^{\tau}\right) w_{q q}^{\tau}+\gamma(-p)\left(3 a_{\lambda}^{-2}(p)+3 a_{\lambda}^{-1}(p) f_{p}^{\tau}+w_{p}^{\tau} f_{p}^{\tau}+w_{p}^{2}\right)\right] v_{p}^{\tau} \\
\equiv & A(\nabla w)\left[v^{\tau}\right]+b^{1}\left(w, w^{\tau}\right) v_{q}^{\tau}+b^{2}\left(w, w^{\tau}\right) v_{p}^{\tau}=0 \quad \text { in } S(\tau),
\end{aligned}
$$

$$
B\left[v^{\tau}\right]=f_{q}^{\tau} v_{q}^{\tau}+(2 g w-\lambda)\left(2 \lambda^{-1 / 2}+f_{p}^{\tau}\right) v_{p}^{\tau}+2 g\left(\lambda^{-1 / 2}+w_{p}\right)^{2} v^{\tau}=0 \quad \text { for } \quad q=0,
$$

where $A(\nabla w)$ is defined in (2.9). Other boundary conditions of $v^{\tau}$ are

$$
\begin{cases}v^{\tau}\left(q, p_{0}\right)=0 & \text { for } \quad-\infty<q<\tau \\ v^{\tau}(\tau, p)=0 & \text { for } \quad p_{0} \leqslant p \leqslant 0 \\ v^{\tau}, \nabla v^{\tau}, \nabla^{2} v^{\tau} \rightarrow 0 & \text { as } \quad q \rightarrow-\infty \text { uniformly for } p .\end{cases}
$$

The boundary condition on the bottom $p=p_{0}$ and on the right side $q=\tau$ are of Dirichlet type and the boundary condition at infinity as $q \rightarrow-\infty$ is of vanishing type. Established in the following section will be that $v^{\tau}$ indeed vanishes exponentially as $q \rightarrow-\infty$.

Proof. Let

$$
\tau^{*}=\sup \left\{\tau^{\#}: v^{\tau}(q, p)>0 \text { in } S(\tau) \text { whenever }-\infty<\tau<\tau^{\#}\right\} .
$$

We first claim that $\tau^{*}$ exists and is finite. Indeed, the result of Proposition 4.6 and Lemma 4.7 is that

$$
w(q, p)= \begin{cases}r_{1}^{+} \phi_{1}(p) e^{-\sqrt{\mu_{1}} q}+O\left(e^{-s_{1} q}\right) & \text { as } \quad q \rightarrow \infty, \\ r_{1}^{-} \phi_{1}(p) e^{\sqrt{\mu_{1}} q}+O\left(e^{s_{1} q}\right) & \text { as } \quad q \rightarrow-\infty,\end{cases}
$$

where $r_{1}^{+}, r_{1}^{-}>0$ and $\sqrt{\mu_{1}}<s_{1}<\min \left(2 \sqrt{\mu_{1}}, \sqrt{\mu_{2}}\right)$. Here, $\mu_{1}$ is the smallest eigenvalue of (4.7) and $\mu_{2}$ is the next smallest eigenvalue; $\phi_{1}$ is an eigenfunction corresponding to $\mu_{1}$. We may assume $\phi_{1}(p)>0$ on the interval $p_{0}<p \leqslant 0$. Proposition 4.6 furthermore asserts that $w_{q}(q, p)>0$ in $S$ for $q$ near $-\infty$ and $w_{q}(q, p)<0$ in $S$ for $q$ near $\infty$. Accordingly, there exists a $\tau^{\#}$ such that if $\tau<\tau^{\#}$ then $v^{\tau}(q, p)>0$ whenever $q<\tau$ and if $\tau>-\tau^{\#}$ then $v^{\tau}(q, p)<0$ whenever $q<\tau$. This proves the claim.

Our goal is to show that $v^{*}(q, p):=v\left(q, p ; \tau^{*}\right) \equiv 0$ in $S\left(\tau^{*}\right)$. Continuity and the maximum principle assert that either $v^{*} \equiv 0$ or $v^{*}>0$ in $S\left(\tau^{*}\right)$. To argue 
by contradiction, we suppose the latter. It is readily seen that $v^{*}\left(\tau^{*}, 0\right)=0$ is the minimum of $v^{*}(q, p)$ in $\overline{S\left(\tau^{*}\right)}$. The boundary condition of $v^{*}$ on $q=\tau^{*}$ in $(3.3 \mathrm{c})$ implies that

$$
v_{p}^{*}\left(\tau^{*}, 0\right)=0=v_{p p}^{*}\left(\tau^{*}\right) .
$$

We shall show that $v_{q}^{*}\left(\tau^{*}, 0\right)=0$ and $v_{q p}^{*}\left(\tau^{*}, 0\right)=0$. (Thereby, it violates the edge point lemma.)

First, the extremal property of $\tau^{*}$ allows us to choose sequences

$$
\tau^{k} \downarrow \tau^{*} \text { and }\left(q^{k}, p^{k}\right) \in \overline{S\left(\tau^{k}\right)} \text { such that } v\left(q^{k}, p^{k} ; \tau^{k}\right)<0 .
$$

Let us write $v^{k}(q, p)=v\left(q, p ; \tau^{k}\right)$. We may choose $v^{k}\left(q^{k}, p^{k}\right)=\min \frac{}{S\left(\tau^{k}\right)} v^{k}(q, p)$. Our claim is that the negative minimum point $\left(q^{k}, p^{k}\right)$ is attained on the smooth part of the top boundary, that is, $p_{k}=0$. Note that $v^{k}\left(\tau^{k}, p\right)=0$ for $p_{0} \leqslant p \leqslant 0$. Since $v^{k}\left(q, p_{0}\right)=0$ for $-\infty<q \leqslant \tau^{k}$ the negative minimum point $\left(q^{k}, p^{k}\right)$ of $v^{k}$ must be attained either in the interior of $S\left(\tau^{k}\right)$ or on the smooth part of the top boundary. If $v^{k}(q, 0) \geqslant 0$ for $-\infty<q<\tau^{k}$, on the other hand, the maximum principle would dictate $v^{k}>0$ in $S\left(\tau^{k}\right)$, which contradicts since the minimum of $v^{k}$ in $S\left(\tau^{k}\right)$ is negative. Therefore, $v^{k}\left(q^{k}, 0\right)<0$ for some $-\infty<q^{k}<\tau^{k}$. As a simple consequence of minimum, $v_{q}^{k}\left(q^{k}, 0\right)=0$.

Next, we claim that $\left\{\left(q^{k}, 0\right)\right\}$ is bounded below. The asymptotic description of $w(q, p)$ in $(3.5)$ restricted to $p=0$ reads

$$
v^{\tau}(q, 0)=w^{\tau}(q, 0)-w(q, 0) \sim r(\tau) e^{\sqrt{\mu_{1}} q} \quad \text { as } q \rightarrow-\infty,
$$

where $r(\tau)=\phi_{1}(0)\left(r_{1}^{+} e^{-2 \tau \sqrt{\mu_{1}}}-r_{1}^{-}\right)$. Since $v^{*}>0$ in $S\left(\tau^{*}\right)$, Lemma 4.7 asserts that $r\left(\tau^{*}\right)>0$. Continuity then yields that $r(\tau)>0$ for $\tau<\tau^{*}+\delta$, where $\delta>0$ is sufficiently small. Accordingly, $v^{\tau}(q, 0)>0$ when $q$ near $-\infty$ for $\tau<\tau^{*}+\delta$. Therefore, a negative minimum point $\left(q^{k}, 0\right)$ of $v^{k}(q, p)$ is bounded below for $k$ large enough so that $\tau^{k}<\tau^{*}+\delta$.

Next, the boundedness of $\left\{\left(q^{k}, 0\right)\right\}$ allows us to take the limit $\left(q^{k}, 0\right) \rightarrow\left(q^{*}, 0\right) \in$ $\partial S\left(\tau^{*}\right)$ as $k \rightarrow \infty$. By continuity, $v_{q}^{*}\left(q^{*}, 0\right)=0$. Furthermore, $\left(q^{*}, 0\right)$ is the corner point $\left(\tau^{*}, 0\right)$. Suppose on the contrary that $-\infty<q^{*}<\tau^{*}$. Since $v^{*}\left(q^{*}, 0\right)=0=$ $v_{q}^{*}\left(q^{*}, 0\right)$ the boundary condition $(3.3 \mathrm{~b})$ at $\left(q^{*}, 0\right)$ reduces to

$$
(2 g w-\lambda)\left(2 \lambda^{-1 / 2}+f_{p}^{\tau}\right) v_{p}^{*}=0 .
$$

On the other hand, since $2 g w-\lambda<0$ and $\left|(2 g w-\lambda)\left(\lambda^{-1 / 2}+v_{p}\right)\right|, \mid(2 g w-\lambda)\left(\lambda^{-1 / 2}+\right.$ $\left.v_{p}^{\tau}\right) \mid>0$, it follows that $v_{p}^{*}\left(q^{*}, 0\right)=0$. This however contradicts the Hopf boundary lemma and thus proves the assertion. Therefore,

$$
v_{q}^{*}\left(\tau^{*}, 0\right)=0
$$

In order to show that $v_{q p}^{*}\left(\tau^{*}, 0\right)=0$, we differentiate the boundary condition of (2.6) and evaluate it at $\left(\tau^{*}, 0\right)$ to obtain

$$
2(2 g w-\lambda)\left(\lambda^{-1 / 2}+v_{p}\right) w_{q p}=0
$$

This uses that $w_{q}\left(\tau^{*}, 0\right)=-\frac{1}{2} v_{q}^{*}\left(\tau^{*}, 0\right)=0$. As in the previous step, since $\mid(2 g w-$ $\lambda)\left(\lambda^{-1 / 2}+w_{p}\right) \mid>0$ it then follows that $w_{q p}\left(\tau^{*}, 0\right)=-\frac{1}{2} v_{q p}^{*}\left(\tau^{*}, 0\right)=0$.

Finally, we use (3.3a) to express $v_{q q}^{*}$ in terms of the other derivatives of order 1 and 2. Since $v_{q}^{*}\left(\tau^{*}, 0\right)=0=v_{q p}^{*}\left(\tau^{*}, 0\right)$, by $(3.6)$ it follows that $v_{q q}^{*}\left(\tau^{*}, 0\right)=0$. This 
however contradicts the edge point lemma ( [17, Lemma 1], [7, Lemma S $]$ ) and proves that $v^{*} \equiv 0$ in $S\left(\tau^{*}\right)$. This proves the symmetry assertion (3.1).

For $\tau<\tau^{*}$, the definition of $\tau^{*}$ in (3.4) ensures that $v^{\tau}(q, p)>0$ in $S(\tau)$. Since $v^{\tau}(\tau, p)=0$ is a minimum of the function $v^{\tau}(q, p)$ in $\overline{S(\tau)}$, the Hopf boundary lemma implies that

$$
w_{q}(\tau, p)=-\frac{1}{2} v_{q}^{\tau}(\tau, p)>0 \quad \text { for } \tau<\tau^{*} \text { and } p_{0}<p<0 .
$$

By continuity, $w_{q}(\tau, 0) \geqslant 0$. To state $(3.2)$, we need to show that $w_{q}(\tau, 0)>0$. If $w_{q}(\tau, 0)=0$, differentiation of the boundary condition of (2.6) yields that

$$
2(2 g w-\lambda)\left(\lambda^{-1 / 2}+w_{p}\right) w_{q p}=0 \quad \text { at }(\tau, 0) .
$$

As before, since $\left|2(2 g w-\lambda)\left(\lambda^{-1 / 2}+w_{p}\right)\right|>0$ this implies $w_{q p}(\tau, 0)=0$. Note that, on the other hand, the linear elliptic differential equation $L\left[v^{\tau}\right]=0$ holds and that $v^{\tau}(\tau, 0)=0$ is a minimum of the function $v^{\tau}(q, p)$ in $\overline{S(\tau)}$. Since

$$
v_{q}^{\tau}(\tau, 0)=-2 w_{q}(\tau, 0)=0, \quad v_{q p}^{\tau}(\tau, 0)=-2 w_{q p}(\tau, 0)=0,
$$

this violates the edge point lemma. Therefore, $w_{q}(\tau, 0)>0$.

This completes the proof.

Our conclusion, in the special case of zero vorticity, partly recovers the well-known result in [4] that any irrotational solitary wave with a supercritical value of parameter is of positive elevation, symmetric and monotone about a single crest, and exponentially decays at infinity to a constant level of depth. A main difference in our statement of result from that in [4] is that the positivity of free-surface elevation is a hypothesis in Theorem 3.1 while, in the irrotational setting, it is a conclusion of an a priori estimate. An interesting unresolved issue is the elevation property of supercritical solitary waves for a general class of nontrivial vorticities. The proof in case of zero vorticity [4] uses the comparison principle with a linear function.

Remark 3.2. Our symmetry result holds for a general class of nonlinear elliptic problems in domains which possess translational symmetry. In particular, the argument as in the proof of Theorem 3.1 applies to a bounded domain proves [12] the analogous symmetry and monotonicity results for periodic water-waves with vorticity under the conditions that each streamline has its minimum below the wave trough within one wavelength and that the wave profile is monotone locally near the trough.

For a general class of vorticities, Constantin and Strauss [3] constructed a global connected branch of periodic water-waves which contains a local curve of (smallamplitude) periodic solutions equipped with the above-mentioned streamline pattern. A simple maximum principle argument then asserts that such a streamline pattern preserves along a connected branch of solutions. Hence, any periodic water-wave on the solution branch obtained in [3] will inherit the same streamline pattern as that of small-amplitude waves on the local curve, and consequently, it is symmetric and monotone. That is to say, for an arbitrary vorticity distribution periodic-wave solutions constructed in [3] are symmetric and monotone. 


\section{Asymptotic description of solutions}

This section provides with exponential decay estimates of solutions to (2.6) and their asymptotic description as $|q| \rightarrow \infty$. The behavior of solutions as $|q| \rightarrow \infty$ is crucial in the symmetrization analysis in Theorem 3.1. Indeed, control of the asymptotic behavior of solutions at infinity is needed to start the method of moving planes. Moreover, asymptotic decay properties of solitary-wave solutions to a nonlinear elliptic problem are of independent interest. The approach taken here is related to that in $[5]$.

Our proof of the exponential decay estimate and the asymptotic expansion of solutions of the nonlinear problem (2.6) hinge on the estimate of the corresponding linear problem. To this end, let us write (2.6) as

$$
\begin{cases}a_{\lambda}^{-2}(p) w_{q q}+a_{\lambda}^{-3}(p)\left(a_{\lambda}^{3}(p) w_{p}\right)_{p}=f_{1}\left(\nabla w, \nabla^{2} w\right) & \text { in } S \\ 2 \lambda^{1 / 2} w_{p}-2 g \lambda^{-1} w=f_{2}(w, \nabla w) & \text { for } p=0 \\ w=0 & \text { for } p=p_{0}\end{cases}
$$

where

$$
\begin{aligned}
f_{1}\left(\nabla w, \nabla^{2} w\right)= & \left(2 a_{\lambda}^{-1}(p)+w_{p}\right) w_{p} w_{q q}-2\left(a_{\lambda}^{-1}(p)+w_{p}\right) w_{q p}+w_{q}^{2} w_{p p} \\
& +\gamma(-p)\left(-a_{\lambda}^{-3}(p) w_{q}^{2}+3 a_{\lambda}^{-1}(p) w_{p}^{2}+w_{p}^{3}\right), \\
f_{2}(w, \nabla w)= & w_{q}^{2}+4 g \lambda^{-1 / 2} w w_{p}+2 g w w_{p}^{2}-\lambda w_{p}^{2} .
\end{aligned}
$$

Observe that $f_{1}\left(\nabla w, \nabla^{2} w\right)$ and $f_{2}(w, \nabla w)$ consist of quadratic or cubic polynomial expressions in their arguments. The system (4.1) is supplemented with the vanishing condition at infinity

$$
w, \nabla w, \nabla^{2} w \rightarrow 0 \quad \text { as } \quad|q| \rightarrow \infty .
$$

It is straightforward to show that the differential operator $a_{\lambda}^{-2}(p) \partial_{q}^{2}+a_{\lambda}^{-3}(p) \partial_{p}\left(a_{\lambda}^{3}(p) \partial_{p}\right)$ in the strip $S$ with the boundary conditions

$$
2 \lambda^{1 / 2} w_{p}-2 g \lambda^{-1} w=0 \quad \text { for } \quad p=0, \quad w=0 \quad \text { for } \quad p=p_{0}
$$

has only continuous spectrum $\left[\mu_{1}, \infty\right)$, where $\mu_{1}=\mu_{1}(\lambda)$ is determined below in Lemma 4.1. We shall show that $\mu_{1}(\lambda)>0$ for a supercritical value of parameter, that is, $\lambda>\lambda_{c}$. The beginning of the continuous spectrum $\mu_{1}$ gives the decay rate of solutions of (4.1). Indeed, if $\mu_{1}>0$ then solutions decay as $\exp \left(-\sqrt{\mu_{1}}|q|\right)$ as $|q| \rightarrow \infty$.

4.1. Exponential decay estimates. It is convenient to state our exponential decay estimates in terms of weighted Hölder norms. Let us denote by $\sigma=\sigma(q)$ the weight function. For a positive integer $k$, a constant $\alpha \in(0,1)$ and for a function $w(q, p)$ defined in $S$, let

$$
\begin{aligned}
&\|w\|_{C_{\sigma}^{k+\alpha}(\bar{S})}=\max \left(\max _{0 \leqslant l_{1}+l_{2} \leqslant k}\right. \sup _{(q, p) \in \bar{S}}\left|\partial_{q}^{l_{1}} \partial_{p}^{l_{2}} w(q, p) \cosh \sigma(q)\right|, \\
&\left.\max _{l_{1}+l_{2}=k} \sup _{(q, p) \in \bar{S}}\left[\partial_{q}^{l_{1}} \partial_{p}^{l_{2}} w(q, p)\right]_{\alpha} \cosh \sigma(q)\right),
\end{aligned}
$$


where the Hölder quotient is defined as

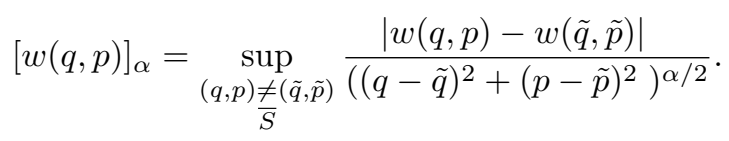

This notion is extended in an obvious way to the case when $\alpha=0$ and to functions of a single variable.

The weight function $\sigma$ is taken to be nonnegative, even, increasing for $q \geqslant 0$, and sublinear, i.e., $\sigma(q+\tilde{q}) \leqslant \sigma(q)+\sigma(\tilde{q})$. Examples include $\sigma(q)=\mu|q|$ with $\mu>0$. If $\sigma(q)$ is linear for $q \geqslant 0$ then $\|w\|_{C_{\sigma}^{k+\alpha}(\bar{S})}<\infty$ implies that $w(q, p)$ and its first $k$ derivatives decay exponentially as $|q| \rightarrow \infty$ uniformly for $p$.

Our first task is to establish an exponential decay estimate for the linear boundary value problem

$$
\begin{cases}a_{\lambda}(p) w_{q q}+\left(a_{\lambda}^{3}(p) w_{p}\right)_{p}=f_{1} & \text { in } S \\ \lambda^{3 / 2} w_{p}-g w=f_{2} & \text { for } p=0 \\ w=0 & \text { for } p=p_{0}\end{cases}
$$

where $f_{1}$ and $f_{2}$ tend to zero as $|q| \rightarrow \infty$ so that improper integrals involving $f_{1}$ and $f_{2}$ converge.

The proof uses an estimate of solutions written by means of the Green's function. Set $f_{1}, f_{2} \equiv 0$, and by the separation of variables $w(q, p)=\Phi(q) \Psi(p)$ the homogeneous problem of $(4.3)$ is written as

$$
\ddot{\Phi}-\mu \Phi=0 \quad \text { for } \quad-\infty<q<\infty,
$$

and

$$
\left\{\begin{array}{l}
\left(a_{\lambda}^{3} \Psi^{\prime}\right)^{\prime}+\mu a_{\lambda} \Psi=0 \text { for } p_{0}<p<0 \\
\lambda^{3 / 2} \Psi^{\prime}(0)-g \Psi(0)=0 \text { and } \Psi\left(p_{0}\right)=0 .
\end{array}\right.
$$

Here and elsewhere, the dot above a variable denotes the differentiation in the $q$ variable and the prime denotes the differentiation in the $p$-variable. It is standard that the Green's function for (4.4) is given by

$$
G_{1}(q ; \mu)=-\frac{1}{\sqrt{\mu}} e^{-\sqrt{\mu}|q|} .
$$

The next lemma is useful for the construction of the Green's function of (4.5).

Lemma 4.1. For $\lambda>\lambda_{c}$, the system

$$
\begin{cases}-\left(a_{\lambda}^{3}(p) v^{\prime}\right)^{\prime}=\mu a_{\lambda}(p) v & \text { for } p_{0}<p<0 \\ \lambda^{3 / 2} v^{\prime}(0)-g v(0)=0, & v\left(p_{0}\right)=0\end{cases}
$$

has an infinite sequence of eigenvalues $\left\{\mu_{n}\right\}$ such that $0<\mu_{1}<\mu_{2}<\cdots<\mu_{n}<\cdots$ and $\lim _{n \rightarrow \infty} \mu_{n}=\infty$. To each $\mu_{n}$ is associated an eigenfunction $\phi_{n}(p)$ which has exactly $n-1$ zeros on the interval $p_{0}<p<0$. We may choose $\phi_{1}(p)>0$ on $p_{0}<p \leqslant 0$. 
Proof. We show that $\mu_{1}(\lambda)>0$ when $\lambda>\lambda_{c}$. The Rayleigh principle states that the smallest eigenvalue of (4.7) is characterized by

$$
\mu_{1}(\lambda)=\inf _{\substack{v \in H^{1}\left(\left(p_{0}, 0\right)\right) \\ v\left(p_{0}\right)=0}} \frac{-g v^{2}(0)+\int_{p_{0}}^{0} a_{\lambda}^{3}(p)\left(v^{\prime}\right)^{2} d p}{\int_{p_{0}}^{0} a_{\lambda}(p) v^{2} d p} .
$$

Note that $\mu_{1}=\mu_{1}(\lambda)$ is a $C^{1}$ function of $\lambda$. From a variational consideration, $\mu_{1}\left(\lambda_{c}\right)=$ 0 ; see [13, Lemma 4.1]. Since the mapping $\lambda \mapsto \mu_{1}(\lambda)$ is strictly increasing whenever $\mu_{1} \leqslant 0$ ( [3, Lemma 3.4]), it then follows that $\mu_{1}(\lambda)>0$ for $\lambda>\lambda_{c}$. Other assertions follow from the standard theory of Sturm-Liouville systems ( [10], for instance). This completes the proof.

Let $v_{1}=v_{1}(p ; \mu)$ and $v_{2}=v_{2}(p ; \mu)$ be two solutions of $-\left(a_{\lambda}^{3}(p) v^{\prime}\right)^{\prime}=\mu a_{\lambda}(p) v$ for $p_{0}<p<0$ satisfying

$$
\begin{cases}v_{1}\left(p_{0} ; \mu\right)=0, & v_{1}^{\prime}\left(p_{0} ; \mu\right)=-1, \\ v_{2}(0 ; \mu)=\frac{\lambda^{3 / 2}}{\sqrt{\lambda^{3}+g^{2}}}, & v_{2}^{\prime}(0 ; \mu)=\frac{g}{\sqrt{\lambda^{3}+g^{2}}},\end{cases}
$$

respectively. The Green's function of (4.7) is then given by

$$
G_{2}(p, \tilde{p} ; \mu)=\left\{\begin{array}{lll}
W(\mu)^{-1} v_{1}(p ; \mu) v_{2}(\tilde{p} ; \mu) & \text { if } \quad p<\tilde{p} \\
W(\mu)^{-1} v_{1}(\tilde{p} ; \mu) v_{2}(p ; \mu) & \text { if } \quad p>\tilde{p}
\end{array}\right.
$$

where

$$
W(\mu)=W\left(v_{1}(\mu), v_{2}(\mu)\right)=\lambda^{3 / 2}\left|\begin{array}{ll}
v_{1}(0 ; \mu) & v_{1}^{\prime}(0 ; \mu) \\
v_{2}(0 ; \mu) & v_{2}^{\prime}(0 ; \mu)
\end{array}\right|
$$

denotes the Wronskian. Standard theory of Sturm-Liouville system [10, Section 8.5] asserts that $G_{2}(p, \tilde{p} ; \mu)$ is a meromorphic function of $\mu$ for each fixed $p$ and $\tilde{p}$ and that $G_{2}(\mu)$ has a simple pole at each $\mu=\mu_{n}$. These are the only poles of $G_{2}(\mu)$. Moreover, the residue of $G_{2}(\mu)$ at $\mu=\mu_{n}$ is $\phi_{n}(p) \phi_{n}(\tilde{p})$.

Finally, on the basis of a method due to Titchmarsh [20, Chapter 15] the Green's function for (4.4)-(4.5) is constructed by means of a contour integral in terms of $G_{1}$ and $G_{2}$ as

$$
G(q, p, \tilde{p})=\sum_{n=1}^{\infty} \frac{1}{\sqrt{\mu_{n}}} e^{-\sqrt{\mu_{n}}|q|} \phi_{n}(p) \phi_{n}(\tilde{p}) .
$$

Accordingly, the solution $w$ of (4.3) is expressed in the convolution form as

$$
w(q, p)=\int_{-\infty}^{\infty} \int_{p_{0}}^{0} G(q-\tilde{q}, p, \tilde{p}) f_{1}(\tilde{q}, \tilde{p}) d \tilde{q} d \tilde{p}-\int_{-\infty}^{\infty} G(q-\tilde{q}, p, 0) f_{2}(\tilde{q}) d \tilde{q} .
$$

We now state our preliminary exponential decay result.

Lemma 4.2. If $\lambda>\lambda_{c}$ and $w \in C^{k+\alpha}(\bar{S})$, where $k \geqslant 2$ is an integer and $\alpha \in(0,1)$, is a solution of (4.3) then $w(q, p)$ decays exponentially as $|q| \rightarrow \infty$. More precisely, the inequality

$$
\|w\|_{C_{\sigma}^{k+\alpha}(\bar{S})} \leqslant C\left(\left\|f_{1}\right\|_{C_{\sigma}^{k-2+\alpha}(\bar{S})}+\left\|f_{2}\right\|_{C_{\sigma}^{k-1+\alpha}(\mathbb{R})}\right)
$$

holds, where $C>0$ is independent of $w$. The weight function satisfies $0<\sigma(q)<$ $\left(\sqrt{\mu_{1}}-\delta\right)|q|$, where $\delta>0$ arbitrary. 
Proof. The proof involves splitting the Green's function $G(q, p, \tilde{p})$ in (4.9) into two pieces. Let

$$
G(q, p, \tilde{p})=\chi(q) G(q, p, \tilde{p})+(1-\chi(q)) G(q, p, \tilde{p}):=G_{b}(q, p, \tilde{p})+G_{u}(q, p, \tilde{p}),
$$

where $\chi$ is a smooth function with support on $[-2,2]$ and $\chi \equiv 1$ on $[-1,1]$. Note that $G_{b}$ is a singular kernel with compact support and $G_{u}$ is of $C^{\infty}$. The proof studies the $C^{0}$-norms of

$$
\begin{aligned}
& w(q, p) \cosh \sigma(q) \\
& =\iint_{S} G_{b}(q-\tilde{q}, p, \tilde{p}) f_{1}(\tilde{q}, \tilde{p}) d \tilde{p} d \tilde{q} \cosh \sigma(q)+\iint_{S} G_{u}(q-\tilde{q}, p, \tilde{p}) f_{1}(\tilde{q}, \tilde{p}) d \tilde{p} d \tilde{q} \cosh \sigma(q) \\
& -\int_{-\infty}^{\infty} G_{b}(q-\tilde{q}, p, 0) f_{2}(\tilde{q}) d \tilde{q} \cosh \sigma(q)-\int_{-\infty}^{\infty} G_{u}(q-\tilde{q}, p, 0) f_{2}(\tilde{q}) d \tilde{q} \cosh \sigma(q),
\end{aligned}
$$

of its derivatives and of $[w(q, p)]_{\alpha} \cosh \sigma(q)$.

In order to estimate terms containing $G_{b}$, let us introduce the partition of unity $\left\{\chi_{j}\right\}$ of $\mathbb{R}$, where each $\chi_{j}$ is supported on the interval $(j-1, j+1)$, and write $f_{1}(q, p)=$ $\sum_{j} f_{1 j}(q, p)$, where $f_{1 j}(q, p)=\chi_{j}(q) f_{1}(q, p)$. Accordingly, $G_{b} * f_{1}=\sum_{j} G_{b} * f_{1 j}$, where each $G_{b} * f_{1 j}$ is supported on $(j-3, j+3)$. A classical result of singular integral operators asserts that the inequality

$$
\left\|G_{b} * f_{1 j}\right\|_{C^{0}(\bar{S})} \leqslant C\left\|f_{1 j}\right\|_{C^{0}(\bar{S})}
$$

holds for each $j$ independently of $j$, whence the inequalities

$$
\begin{aligned}
\left\|G_{b} * f_{1}\right\|_{C_{\sigma}^{0}(\bar{S})} & =\left\|\left(G_{b} * f_{1}\right) \cosh \sigma\right\|_{C^{0}(\bar{S})}=\left\|\sum_{j}\left(G_{b} * f_{1 j}\right) \cosh \sigma\right\|_{C^{0}(\bar{S})} \\
& \leqslant 6 \sup _{j}\left\|\left(G_{b} * f_{1 j}\right) \cosh \sigma\right\|_{C^{0}(\bar{S})} \leqslant 6 C \sup _{j}\left\|f_{1 j} \cosh \sigma\right\|_{C^{0}(\bar{S})} \\
& \leqslant 6 C \sup \left\|f_{1} \cosh \sigma\right\|_{C^{0}(\bar{S})}
\end{aligned}
$$

hold. The estimate of a Hölder quotient of $G_{b} * f_{1}$ is nearly identical. A standard regularity result for singular integral operators then asserts that the inequality

$$
\left\|G_{b} * f_{1}\right\|_{C_{\sigma}^{k+\alpha}(\bar{S})} \leqslant C\left\|f_{1}\right\|_{C_{\sigma}^{k-2+\alpha}(\bar{S})}
$$

holds for $k \geqslant 2$, where $C>0$ is independent of $f_{1}$. An analogous inequality can be established for the boundary term $\int G_{b}(q-\tilde{q}, p, 0) f_{2}(\tilde{q}) d \tilde{q} \cosh \sigma(q)$.

Next is the estimate of the contribution of the terms containing $G_{u}$. By the subadditivity property of $\sigma$ it follows that [4, Lemma 3.5]

$$
\begin{aligned}
& \left|\iint_{S} G_{u}(q-\tilde{q}, p, \tilde{p}) f_{1}(\tilde{q}, \tilde{p}) d \tilde{p} d \tilde{q} \cosh \sigma(q)\right| \\
& \leqslant\left|\iint_{S} G_{u}(q-\tilde{q}, p, \tilde{p}) f_{1}(\tilde{q}, \tilde{p}) \cosh \sigma(\tilde{q}) \cosh \sigma(q-\tilde{q}) d \tilde{p} d \tilde{q}\right| \cdot \sup \left|\frac{\cosh \sigma(q)}{\cosh \sigma(\tilde{q}) \cosh \sigma(q-\tilde{q})}\right| \\
& \leqslant 4\left|\iint_{S} G_{u}(q-\tilde{q}, p, \tilde{p}) f_{1}(\tilde{q}, \tilde{p}) \cosh \sigma(\tilde{q}) \cosh \sigma(q-\tilde{q}) d \tilde{p} d \tilde{q}\right| \cdot
\end{aligned}
$$


Observe that $G_{u}$ is bounded above by an exponential function. More precisely, it satisfies the asymptotics [18, Lemma 5]

$$
G_{u}(q, p, \tilde{p})=\frac{1}{\sqrt{\mu_{1}}} e^{-\sqrt{\mu_{1}}|q|} \phi_{1}(p) \phi_{1}(\tilde{p})+A(q, p, \tilde{p}) e^{-\sqrt{\mu_{2}}|q|} \quad \text { as } \quad|q| \rightarrow \infty,
$$

where $A(q, p, \tilde{p})$ is bounded and smooth. Similar expansions may be established for derivatives of $G_{u}$. Accordingly,

$$
\begin{aligned}
& \left|\iint_{S} G_{u}(q-\tilde{q}, p, \tilde{p}) f_{1}(\tilde{q}, \tilde{p}) \cosh \sigma(\tilde{q}) \cosh \sigma(q-\tilde{q}) d \tilde{p} d \tilde{q}\right| \\
& \quad=\left|\iint_{S} G_{u}(\tilde{q}, p, \tilde{p}) \cosh \sigma(\tilde{q}) d \tilde{p} d \tilde{q}\right|\left\|f_{1} \cosh \sigma\right\|_{C^{0}(\bar{S})} \leqslant C\left\|f_{1}\right\|_{C_{\sigma}^{0}(\bar{S})}
\end{aligned}
$$

provided that $0<\sigma(q)<\left(\sqrt{\mu_{1}}-\delta\right)|q|$, where $\delta>0$ is arbitrarily small. The estimate for the Hölder quotient is straightforward, and the inequalities

$$
\begin{aligned}
& \left|\frac{\left(G_{u} * f_{1}\right)(q, p)-\left(G_{u} * f_{1}\right)(\tilde{q}, \tilde{p})}{\left((q-\tilde{q})^{2}+(p-\tilde{p})\right)^{\alpha / 2}} \cosh \sigma(q)\right| \\
& \leqslant\left|\iint_{S} G_{u}(q-\tilde{q}, p, \tilde{p}) \frac{\cosh \sigma(q)}{\cosh \sigma(\tilde{q})}\left[f_{1}(q, p)\right]_{\alpha} \cosh \sigma(q)\right| \\
& \leqslant C \sup _{S}\left(\left[f_{1}(q, p)\right]_{\alpha} \cosh \sigma(q)\right)
\end{aligned}
$$

hold, where $C>0$ is independent of $f_{1}$. Standard elliptic regularity theory [8] then asserts that

$$
\left\|G_{u} * f_{1}\right\|_{C_{\sigma}^{k+\alpha}(\bar{S})} \leqslant C\left\|f_{1}\right\|_{C_{\sigma}^{k-2+\alpha}(\bar{S})}
$$

for $k \geqslant 2$ an integer, where $C>0$ is independent of $f_{1}$. The estimate for the boundary term is exactly the same. This completes the proof.

An analogous decay estimate for (4.3) with the inhomogeneous Dirichlet boundary condition on $p=0$ will be useful in future considerations.

Lemma 4.3. If $\lambda>\lambda_{c}$ and $w \in C^{k+\alpha}(\bar{S}), k \geqslant 2$ an integer and $\alpha \in(0,1)$, is a solution of

$$
\left\{\begin{array}{l}
a_{\lambda}(p) w_{q q}+\left(a_{\lambda}^{3}(p) w_{p}\right)_{p}=f_{1} \text { in } S \\
w=f_{2} \quad \text { for } p=0 \text { and } w=0 \text { for } p=p_{0}
\end{array}\right.
$$

then the inequality

$$
\|w\|_{C_{\sigma}^{k+\alpha}(\bar{S})} \leqslant C\left(\left\|f_{1}\right\|_{C_{\sigma}^{k-2+\alpha}(\bar{S})}+\left\|f_{2}\right\|_{C_{\sigma}^{k-1+\alpha}(\mathbb{R})}\right)
$$

holds, where $C>0$ is independent of $w$. The weight function satisfies $0<\sigma(q)<$ $\left(\sqrt{\nu_{1}}-\delta\right)|q|$ for $\delta>0$ arbitrarily small, where $0<\sqrt{\mu_{1}}<\sqrt{\nu_{1}} / 2<\sqrt{\nu_{1}}$.

Proof. The proof is identical to that of Lemma 4.2 except that the Green's function of the homogeneous problem of (4.13) is given by

$$
G^{D}(q, p, \tilde{p})=\sum_{n=1}^{\infty} \frac{1}{\sqrt{\nu_{n}}} e^{-\sqrt{\nu_{n}}|q|} \phi_{n}^{D}(p) \phi_{n}^{D}(\tilde{p}) .
$$


Here, $\nu_{n}$ and $\phi_{n}^{D}$ are determined such that the Sturm-Liouville system

$$
\left\{\begin{array}{l}
-\left(a_{\lambda}^{3}(p) v^{\prime}\right)^{\prime}=\nu a_{\lambda}(p) v \quad \text { for } \quad p_{0}<p<0 \\
v(0)=0, \quad v\left(p_{0}\right)=0
\end{array}\right.
$$

admits an infinite sequence of eigenvalues $0<\nu_{1}<\nu_{2}<\cdots<\nu_{n}<\cdots$ with $\lim _{n \rightarrow \infty} \nu_{n}=\infty$ and to each $\nu_{n}$ is associated an eigenfunction $\phi_{n}^{D}$. Moreover, $0<$ $\sqrt{\mu_{1}}<\sqrt{\nu_{1}} / 2$, where $\mu_{1}$ is the smallest eigenvalue of (4.7).

The weighted estimate of the linear problem (4.3) in Lemma 4.2 leads to that the solutions of the nonlinear problem (4.1), which decays to zero as $|q| \rightarrow \infty$ do so at an exponential rate. The idea is that since the nonlinear terms in (4.1) are composed of quadratic or cubic polynomial expressions if a solution decays at a certain rate then the nonlinear terms decay at a faster rate.

The theorem below states that the solutions of (4.1)-(4.2) as $|q| \rightarrow \infty$ have the exponential decay rate of the corresponding linear problem.

Theorem 4.4. If $\lambda>\lambda_{c}$ and $w \in C^{k+\alpha}(\bar{S})$, where $k \geqslant 2$ is an integer and $\alpha \in(0,1)$, is a solution to (4.1)-(4.2), then

$$
\|w\|_{C_{\sqrt{\mu_{1}|q|}}^{k+\alpha}(\bar{S})}<\infty
$$

where $\mu_{1}>0$ is the smallest eigenvalue of (4.7) in Lemma 4.1.

Proof. Since the nonlinear terms $f_{1}\left(\nabla w, \nabla^{2} w\right)$ and $f_{2}(w, \nabla w)$ behave at least quadratically simple estimates

$$
\begin{aligned}
\left\|f_{1}\left(\nabla w, \nabla^{2} w\right)\right\|_{C_{2 \sigma}^{k-2+\alpha}(\bar{S})} & \leqslant C\|w\|_{C_{\sigma}^{k+\alpha}(\bar{S})}^{2}, \\
\left\|f_{2}(w, \nabla w)\right\|_{C_{2 \sigma}^{k-1+\alpha}(\mathbb{R})} & \leqslant C\|w\|_{C_{\sigma}^{k+\alpha}(\mathbb{R})}^{2}
\end{aligned}
$$

hold, where $C>0$ is independent of $w$. Combining it with the linear estimate (4.11) then yields that

$$
\|w\|_{C_{\sigma}^{k+\alpha}(\bar{S})} \leqslant C\|w\|_{C_{\sigma / 2}^{k+\alpha}(\bar{S})}^{2}
$$

holds for any $0<\sigma(q)<\left(\sqrt{\mu_{1}}-\delta\right)|q|$, where $\delta>0$ is arbitrary. Let us consider the class of "admissible" weight functions $\sigma(q)$ such that $\|w\|_{C_{\sigma}^{k+\alpha}(\bar{S})}<\infty$. The estimate (4.17) says that if $\sigma(q) / 2 \in R$ then $\min \left(\sigma(q),\left(\sqrt{\mu_{1}}-\delta\right)|q|\right) \in R$, doubling the decay rate up to $\sigma(q)=\left(\sqrt{\mu_{1}}-\delta\right)|q|$.

In order to obtain the exact decay rate without $\delta$, we write the solution $w$ of (2.6) via the Fourier transform in the $q$-variable as

$$
w(q, p)=\frac{1}{\sqrt{2 \pi}} \int_{-\infty}^{\infty} e^{i q \xi}\left(\int_{p_{0}}^{0} \hat{G}(\xi, p, \tilde{p}) a_{\lambda}^{3}(\tilde{p}) \hat{f}_{1}(\xi, \tilde{p}) d \tilde{p}-\hat{G}(\xi, p, 0) \frac{1}{2} \lambda \hat{f}_{2}(\xi)\right) d \xi .
$$

Here and elsewhere, the hat above a variable denotes the Fourier transform in the $q$-variable. The nonlinear inhomogeneous terms $f_{1}(q, p)=f_{1}\left(\nabla w(q, p), \nabla^{2} w(q, p)\right)$ and $f_{2}(q)=f_{2}(w(q, 0), \nabla w(q, 0))$ are treated as functions of $q$ and $p$ variables.

Note that $w(q, p)$ decays exponentially like $\exp (-\mu|q|)$ as $|q| \rightarrow \infty$ if and only if $\hat{w}(\xi, p)$ is analytic in the complex strip $|\operatorname{Im} \xi|<\mu$. The estimates (4.16) then imply 
that $\hat{f}_{1}(\xi, \tilde{p})$ and $\hat{f}_{2}(\xi, 0)$ are analytic in the complex strip $|\operatorname{Im} \xi|<2 \sqrt{\mu_{1}}$ uniformly for $\tilde{p}$. Consequently,

$$
\hat{w}(\xi, p)=\int_{p_{0}}^{0} \hat{G}(\xi, p, \tilde{p}) a_{\lambda}^{3}(\tilde{p}) \hat{f}_{1}(\xi, \tilde{p}) d \tilde{p}-\hat{G}(\xi, p, 0) \frac{1}{2} \lambda \hat{f}_{2}(\xi)
$$

is analytic in the largest strip in which $\hat{G}(\xi, p, \tilde{p}) \hat{f}_{1}(\xi, \tilde{p})$ and $\hat{G}(\xi, p, 0) \hat{f}_{2}(\xi)$ are defined, that is, up to the first pole of $\hat{G}(\xi, p, \tilde{p})$ and $\hat{G}(\xi, p, 0)$, and integrable. Recall that the first poles of $\hat{G}(\xi)$ occur at $\xi= \pm i \sqrt{\mu_{1}}$.

On account of (4.12) it follows that

$$
|\hat{G}(\xi, p, \tilde{p})|,|\hat{G}(\xi, p, 0)|<\frac{C}{1+|\xi|^{2}} \quad \text { as }|\xi| \rightarrow \infty,
$$

where $C>0$ is independent of $\xi$. Since $f_{1} \in C^{\alpha}(\bar{S})$ and $f_{2} \in C^{\alpha}(\mathbb{R})$ the inequalities

$$
\left|\hat{G}(\xi, p, \tilde{p}) \hat{f}_{1}(\xi, \tilde{p})\right|,\left|\hat{G}(\xi, p, 0) \hat{f}_{2}(\xi)\right|<\frac{C}{\left(1+|\xi|^{2}\right)^{(1+\alpha / 2)}}
$$

hold true. That is, $\hat{G}(\xi, p, \tilde{p}) \hat{f}_{1}(\xi, \tilde{p})$ and $\hat{G}(\xi, p, 0) \hat{f}_{2}(\xi)$ are integrable in the strip $|\operatorname{Im} \xi|<\sqrt{\mu_{1}}$. This recovers the exact decay rate $\sigma(q)=\sqrt{\mu_{1}}|q|$. The proof is complete.

The analogous result to that of Theorem 4.4 for the boundary-layer problem may be established.

Lemma 4.5. If $\lambda>\lambda_{c}$ and $w \in C^{k+\alpha}(\bar{S})$, where $k \geqslant 2$ is an integer and $\alpha \in(0,1)$, is a solution of

$$
\left\{\begin{array}{l}
a_{\lambda}^{-2}(p) w_{q q}+a_{\lambda}^{-3}(p)\left(a_{\lambda}^{3}(p) w_{p}\right)_{p}=f_{1}\left(\nabla w, \nabla^{2} w\right) \text { in } S \\
w=f_{2} \text { for } p=0 \text { and } w=0 \text { for } p=p_{0}
\end{array}\right.
$$

with the vanishing boundary conditions $w, \nabla w, \nabla^{2} w \rightarrow 0$ as $|q| \rightarrow \infty$, then $w(q, p)$ decays exponentially as $|q| \rightarrow \infty$. That is,

$$
\|w\|_{C_{\nu_{1}|q|}^{k+\alpha}(\bar{S})}<\infty
$$

where $\nu_{1}>0$ is the smallest eigenvalue of (4.15) in Lemma 4.3.

The proof is identical to that of Theorem 4.4 and hence is omitted.

4.2. Asymptotic expansions of solutions. In order to start the method of moving planes in the setting of noncompact domain, one needs more detailed knowledge of the solution behavior at infinity than an estimate of exponential decay. In this subsection, we obtain a rigorous asymptotic expansion of solutions, which provides sufficient control of solutions at infinity in the proof of Theorem 3.1.

Proposition 4.6. If $\lambda>\lambda_{c}$ and $w \in C^{k+\alpha}(\bar{S})$, where $k \geqslant 2$ is an integer and $\alpha \in(0,1)$, is a solution of (4.1)-(4.2) then $w$ satisfies the asymptotic estimate

$$
\left|\partial_{q}^{l_{1}} \partial_{p}^{l_{2}}\left(w(q, p)-r_{1} \phi_{1}(p) e^{-\sqrt{\mu_{1}} q}\right)\right|<C e^{-s_{1} q} \quad \text { as } \quad q \rightarrow \infty
$$

for all $l_{1}+l_{2} \leqslant k-1$ for some constant $r_{1}$. Here, $\mu_{1}$ is the smallest eigenvalue of (4.7) and $\phi_{1}$ is the corresponding eigenfunction. The exponent $s_{1}$ satisfies $\sqrt{\mu_{1}}<$ $s_{1}<\min \left(2 \sqrt{\mu_{1}}, \sqrt{\mu_{2}}\right)$, where $\mu_{2}$ is the second smallest eigenvalue of (4.7). 
Proof. Recall that $w$ is written in terms of the Fourier transform of the Green's function as

$$
w(q, p)=\frac{1}{\sqrt{2 \pi}} \int_{-\infty}^{\infty} e^{i q \xi}\left(\int_{p_{0}}^{0} \hat{G}(\xi, p, \tilde{p}) a_{\lambda}^{3}(\tilde{p}) \hat{f}_{1}(\xi, \tilde{p}) d \tilde{p}-\hat{G}(\xi, p, 0) \frac{1}{2} \lambda \hat{f}_{2}(\xi)\right) d \xi,
$$

where $f_{1}(q, p)=f_{1}\left(\nabla w(q, p), \nabla^{2} w(q, p)\right)$ and $f_{2}(q)=f_{2}(w(q, 0), \nabla w(q, 0))$ are treated as functions of $q$ and $p$. Note that $w(q, p)$ decays exponentially like $\exp (-\mu|q|)$ as $|q| \rightarrow \infty$ if and only if $\hat{w}(\xi, p)$ is analytic in the complex strip $|\operatorname{Im} \xi|<\mu$.

By virtue of Theorem 4.4, it follows that $\|w\|_{C_{\sqrt{\mu_{1}|q|}}^{k+\alpha}(\bar{S})}<\infty$. Estimates (4.16) then assert that

$$
\left\|f_{1}\right\|_{C_{2 \sqrt{\mu_{1}}|q|}^{k-2+\alpha}(\bar{S})},\left\|f_{2}\right\|_{C_{2 \sqrt{\mu_{1}}|q|}^{k-1+\alpha}(\mathbb{R})}<\infty,
$$

which in terms of the Fourier transform implies that $\hat{f}_{1}(\xi, \tilde{p})$ and $\hat{f}_{2}(\xi)$ are analytic in the strip $|\operatorname{Im} \xi|<2 \sqrt{\mu_{1}}$. As is shown in the proof of Theorem 4.4, on the other hand, $\hat{G}(\xi, p, \tilde{p})$ is analytic in $|\operatorname{Im} \xi|<\sqrt{\mu_{1}}$ and it loses analyticity on the $\operatorname{line}|\operatorname{Im} \xi|=\sqrt{\mu_{1}}$ due to the presence of simple poles.

In order to obtain the asymptotic expansion (4.19) of $w(q, p)$ as $q \rightarrow \infty$ we deform the contour integration in (4.20) in the upper half plane of the complex plane. Let us choose $\sqrt{\mu_{1}}<s_{1}<\min \left(2 \sqrt{\mu_{1}}, \sqrt{\mu_{2}}\right)$, that is above $\sqrt{\mu_{1}}$ but within the strip of analyticity of $\hat{G}(\xi, p, \tilde{p})$. The calculus of residues yields that

$$
\begin{aligned}
w(q, p)= & \frac{1}{2 \pi i} e^{-\sqrt{\mu_{1}} \xi}\left(\int_{p_{0}}^{0} \operatorname{res}\left(\hat{G}\left(i \sqrt{\mu_{1}}, p, \tilde{p}\right)\right) a_{\lambda}^{3}(\tilde{p}) \hat{f}_{1}\left(i \sqrt{\mu_{1}}, \tilde{p}\right) d \tilde{p}\right. \\
& \left.-\operatorname{res}\left(\hat{G}\left(i \sqrt{\mu_{1}}, p, 0\right)\right) \frac{1}{2} \lambda \hat{f}_{2}\left(i \sqrt{\mu_{1}}\right)\right) \\
& +\frac{1}{\sqrt{2 \pi}} \int_{-\infty}^{\infty} e^{-s_{1} q} e^{i q \xi}\left(\int_{p_{0}}^{0} \hat{G}\left(\xi+i s_{1}, p, \tilde{p}\right) a_{\lambda}^{3}(\tilde{p}) \hat{f}_{1}\left(\xi+i s_{1}, \tilde{p}\right) d \tilde{p}\right. \\
= & \left.-\hat{G}\left(\xi+i s_{1}, p, 0\right) \frac{1}{2} \lambda \hat{f}_{2}\left(\xi+i s_{1}\right)\right) d \xi \\
= &
\end{aligned}
$$

for some constant $r_{1}$. This uses that $\operatorname{Res}\left(G\left(p, \tilde{p} ; \mu_{1}\right)\right)=\phi_{1}(p) \phi_{1}(\tilde{p})$.

The above residue formula proves the assertion so long as the integrand is absolutely integrable uniformly for $p_{0}<p<0$. Indeed, since

$$
|\hat{G}(\xi, p, \tilde{p})|<\frac{C}{1+|\xi|^{2}} \quad \text { as }|\xi| \rightarrow \infty \text { uniformly for } p \text { and } \tilde{p}
$$

(see the proof of Theorem 4.4) and since $w \in C^{k+\alpha}(\bar{S}), k \geqslant 2$ and $\alpha \in(0,1)$, it follows that

$$
\left|(i \xi)^{l_{1}} \partial_{p}^{l_{2}}\left(\int_{p_{0}}^{0} \hat{G}(\xi, p, \tilde{p}) a_{\lambda}^{3}(\tilde{p}) \hat{f}_{1}(\xi, \tilde{p}) d \tilde{p}-\hat{G}(\xi, p, 0) \frac{1}{2} \lambda \hat{f}_{2}(\xi)\right)\right|
$$

is integrable for any $l_{1}+l_{2} \leqslant k-1$ uniformly in $p_{0} \leqslant p \leqslant 0$. This completes the proof. 
The same calculations carried out on the lower half plane $\operatorname{Im} \xi<0$ leads to an analogous asymptotic estimate as $q \rightarrow-\infty$ :

$$
\left|\partial_{q}^{l_{1}} \partial_{p}^{l_{2}}\left(w(q, p)-r_{1}^{-} \phi_{1}(p) e^{\sqrt{\mu_{1}} q}\right)\right|<C e^{s_{1} q} \quad \text { as } \quad q \rightarrow-\infty
$$

for $l_{1}+l_{2} \leqslant k-1$, where $r_{1}^{-}$is a constant.

Note that if $w(q, p) \geqslant 0$ then $r_{1} \geqslant 0$. Indeed, if $r_{1}<0$ then (4.19) contradicts the vanishing boundary condition as $q \rightarrow \infty$. Moreover, $r_{1}>0$ provided the weak form of positivity of solution.

Lemma 4.7. If a solution $w$ of (4.1)-(4.2) satisfies $w(q, p) \geqslant 0$ in $S$ and $w(q, 0)>0$ for all $q \in \mathbb{R}$ then $r_{1}, r_{1}^{-}>0$.

Proof. Suppose that $r_{1}=0$. One may view $w$ as a solution of (4.18) with $f_{2}(q)=$ $w(q, 0)$ and express it in terms of the Green's function of the Dirichlet problem as

$$
\begin{aligned}
w(q, p)=\int_{-\infty}^{\infty} \int_{p_{0}}^{0} G^{D}(q-\tilde{q}, p, \tilde{p}) a_{\lambda}^{3}(\tilde{p}) f_{1}(\nabla w(\tilde{q}, \tilde{p}), & \left.\nabla^{2} w(\tilde{q}, \tilde{p})\right) d \tilde{p} d \tilde{q} \\
& -\int_{-\infty}^{\infty} G^{D}(q-\tilde{q}, p, 0) w(\tilde{q}, 0) d \tilde{q}
\end{aligned}
$$

Note that $G^{D}(q, p, \tilde{p})$ is positive and decays as $|q| \rightarrow \infty$ like $\exp \left(-\sqrt{\nu_{1}}|q|\right)$. Since $w(q, 0)>0$ it follows that $w(q, p)>O\left(e^{-\sqrt{\nu_{1}}|q|}\right)$. On the other hand, since $r_{1}=0$ the asymptotic description (4.19) would yield that $w(q, p)<O\left(e^{-s_{1}|q|}\right)$ as $q \rightarrow \infty$, where $\sqrt{\mu_{1}}<s_{1}<\min \left(2 \sqrt{\mu_{1}}, \sqrt{\mu_{2}}\right)$ This contradicts since $0<\sqrt{\mu_{1}}<\sqrt{\nu_{1}} / 2$. The proof of $r_{1}^{-}>0$ is the same. This completes the proof.

\section{Acknowledgements}

The author would like to thank the anonymous referee for many valuable suggestions which helped improve the readership of the present manuscript.

\section{References}

[1] C. Amick and J. F. Toland, On solitary water-waves of finite amplitude, Arch. Rational Mech. Anal. 76 (1981) 9-95.

[2] J. T. Beale, The existence of solitary water waves, Comm. Pure Appl. Math. 5 (1977) 237-275.

[3] A. Constantin and W. Strauss, Exact steady periodic water waves with vorticity, Comm. Pure Appl. Math. 57 (2004) 481-27.

[4] W. Craig and P. Sternberg, Symmetry of solitary waves, Comm. P.D.E. 13 (1988) 603-633.

[5] - Symmetry of free-surface flows, Arch. Rational Mech. Anal. 118 (1992) 1-36.

[6] K. O. Friedrichs and D. H. Hyers, The existence of solitary waves, Comm. Pure. Appl. Math. 7 (1954) 517-550.

[7] B. Gidas, W.-M. Ni, and L. Nirenberg, Symmetry and related properties via the maximum principle, Comm. Math. Phys 68 (1979) 209-243.

[8] D. Gilbarg and N. Trudinger, Elliptic Partial Differential Equations of Second Order, Reprint of the 1998 edition, Springer, Berlin (2001).

[9] J. O. Herzog, Phragmén-Lindelöf theorems for second order quasi-linear elliptic partial differential equations, Proc. Amer. Math. Soc. 15 (1964) 721-728.

[10] E. Hille, Lectures on Ordinary Differential Equations, Addison-Wesley (1969).

[11] H. Hopf, Lectures on Differential Geometry in the Large, Stanford University (1956).

[12] V. M. Hur, Symmetry of steady periodic water waves with vorticity, Philos. Trans. R. Soc. Lond. Ser A Math. Phys. Eng. Sci 365 (2007) 2203-2214.

[13] — Exact solitary water waves with vorticity, Arch. Rational Mech. Anal. (2008) In press. 
[14] G. Keady and W. G. Pritchard, Bounds for surface solitary waves, Proc. Cambridge Philos. Soc. 76 (1974) 345-358.

[15] J. Lighthill, Waves in Fluids, Cambridge University Press, Cambridge (1978).

[16] J. B. McLeod, The Froude number for solitary waves, Proc. Roy. Soc. Edinburgh Sect A 97 (1984) 193-197.

[17] J. Serrin, A symmetry property in potential theory, Arch. Rational Mech. Anal. 43 (1971) 304318.

[18] S. M. Sun and S. Shen, A new solitary wave solution for water waves with surface tension, Ann. Mat. Pura Appl. 162 (1992) 179-214.

[19] G. Thomas and G. Klopman, Wave-current interactions in the nearshore region, Gravity Waves in Water of Finite Depth, Advances in Fluid Mechanics 10 (1997) 215-319.

[20] E. C. Titchmarsh, Eigenfunction Expansions Associated with Second-order Differential Equations, Vol. 2, Oxford University Press, New York/London (1964).

Department of Mathematics, Massachusetts Institute of Technology, 77 Massachusetts Avenue, Cambridge, MA 02139, USA

E-mail address: verahur@math.mit.edu 